Revista Signos

2008, 41(67)

$177-202$

\title{
El género manual en las disciplinas académicas: Una caracterización desde el sistema de la obligación*
}

\author{
Rosa María Gutiérrez \\ Pontificia Universidad Católica de Valparaíso \\ Chile
}

Resumen: El manual es un género discursivo cuya función predominante es la de regular la inserción de los aprendices a un área de conocimiento específica. Desde esta perspectiva, se trata de un género que adquiere gran relevancia en el marco de la enseñanza y aprendizaje inicial de una disciplina. Es por esta razón que, en este estudio, se pretende caracterizar el género manual, a partir de la probabilidad de ocurrencia de las configuraciones lexicogramaticales que, en español, realizan modulación obligativa de la cláusula. El contexto de observación está conformado por un corpus correspondiente a los manuales de cuatro áreas disciplinares: Psicología y Trabajo Social (Ciencias Sociales y Humanas); y Química e Ingeniería en Construcción (Ciencias Básicas y de la Ingeniería) (Corpus PUCV-2006). El marco teórico y analítico, por su parte, corresponde a la Gramática Sistémico Funcional, específicamente al sistema de la modulación obligativa. Para explorar el corpus se utiliza el etiquetador El Grial (www.elgrial.cl) y la estimación probabilística se desarrolla utilizando probabilidades condicionadas. Los principales resultados indican que se prefieren utilizar configuraciones directas y congruentes para construir la regulación, lo que pone de manifiesto que la relación entre experto y aprendiz es marcadamente distante. Esto, a su vez, se constituye en una característica del tenor del género manual, asociada a la específica situación comunicativa en la que este género es empleado: la enseñanza.

Palabras Clave: Manual, género discursivo, discurso académico, lingüística sistémico funcional, modulación.

Recibido: 15-VI-2007

Aceptado: 19-XI-2007
* Trabajo enmarcado en el proyecto Fondecyt 1060440, PUCV.

Correspondencia: Rosa María Gutiérrez (rosamaria.gutierrez@ucv.cl). Tel.: (56-32) 227 3378. Instituto de Literatura y Ciencias del Lenguaje, Pontificia Universidad Católica de Valparaíso. Avenida Brasil 2830, piso 9, Valparaíso, Chile. 


\section{The textbook genre in academia disciplines: A characterization from the obligation system}

Abstract: The textbook is a discourse genre whose communicative predominant function is that of regulating the insertion of learners into a specific area of knowledge. From this perspective, it is a genre of great relevance in the context of disciplinary literacy. Taking as context of observation the textbooks of four disciplinary areas: Psychology and Social Work, corresponding to the social and human sciences; and Chemistry and Construction Engineering, corresponding to the basic sciences and those of the engineering (Corpus PUCV-2006) and using as a tool of linguistic analysis the Sistemic Functional Grammar, specifically, the system of obligative modulation; this study pretends to describe and characterize the textbook genre, from the variation that the probabilistic profile of lexicogramatical configurations that, in Spanish, realize obligative modulation of the clause present (Gutiérrez, 2007). For the exploitation of the corpus, we used the tagger El Grial (www.elgrial.cl) that is the one that administers the registers used in this study. The probabilistic estimation is developed through conditioned probabilities and the variation of the system, in the frame of the multiregister corpus is estimated on a global error of $3 \%$. The principal results indicate that in the context of the textbook direct and congruent configurations are preferred to construct regulation, which reveals that the relation between expert and apprentice is distant. In turn, this constitutes a characteristic of the tenor of the genre, associated with the specific communicative situation in which this genre is used: teaching.

Key Words: Text book, academic discourse, systemic functional linguistic, modulation system.

\section{INTRODUCCIÓN}

Un aspecto importante de la formación académica depende de los géneros discursivos a través de los que se nos acerca al conocimiento de una determinada disciplina. A través de ellos, configuramos una identidad en torno a una comunidad discursiva, posibilitando no solo el acercamiento al conocimiento especializado de la misma, sino que también el acceso al modo de negociar la construcción de dicho conocimiento. De allí la importancia de conocer cuáles son esos géneros y qué modos de negociación los caracterizan.

En el marco del proyecto FONDECYT 1060440, hemos recolectado un corpus, PUCV-2006, que reúne todo el material utilizado en cuatro carreras de la Pontificia Universidad Católica de Valparaíso para la enseñanza de la correspondiente disciplina. Este material conforma un corpus de 58.594.630 palabras, el que ha sido clasificado, de una parte, por área disciplinar, dividiéndose en las cuatro disciplinas formantes del corpus: Psicología, Trabajo Social, Ingeniería en Construcción y Química Industrial; y de otra, ha sido clasificado por género discursivo, lo que da origen a nueve géneros: Texto disciplinar, Artículo de investigación científica, Conferencia, Diccionario, Guía didáctica, Informe, Norma, Manual y Test (Parodi, Venegas, Gutiérrez \& Ibáñez, 2008).

En este artículo, abordamos la descripción de uno de estos géneros: el manual. Más allá de lo que implica caracterizar lingüísticamente los géneros utilizados en la enseñanza de una disci- 
plina, lo que mueve la descripción del manual es que se trata del único que se utiliza en las cuatro áreas disciplinares formantes del corpus PUCV-2006. A esta situación, se suma el hecho de que proporciona, tal como lo sostiene Halliday (2004a, 2004b), Cubo de Severino (2005) y Hyland (2004), un primer acercamiento al conocimiento disciplinar, iniciando al estudiante en "las normas, valores, creencias e ideologías de una cultura académica particular" (Cubo de Severino, 2005: 326). En este sentido es que afirmamos que la función comunicativa predominante en el manual es la regulativa (Parodi et al., 2008), esto es, la función de uniformar, normalizar y sistematizar el marco en el cual se asentarán los nuevos conocimientos, guiando de este modo la inserción en la disciplina (o materia específica).

Desde la Gramática Sistémico Funcional (GSF), la función regulativa se asocia al sistema de la interpersonalidad el cual, como su nombre lo indica, dice relación con la interacción de los hablantes en un evento comunicativo; esto es, qué roles desempeñan, cómo los intercambian y qué mecanismos utilizan para negociar significados (Halliday, 1984; Matthiessen, 1995; Martin, Matthiessen \& Painter, 1997; Halliday \& Matthiessen, 2004). En este marco, la regulación es una subfunción asociada a la función mayor de requerir u ordenar, la cual a su vez es parte del sistema de la modulación obligativa. Para este estudio, tal sistema es tanto el marco teórico como el modelo de análisis que se utilizará. Como marco teórico, la gradiente de la obligación, desde la GSF, provee del contexto para la interpretación de los resultados; como modelo de análisis, el sistema presenta las estructuras o configuraciones lexicogramaticales que rastreamos en los manuales de nuestro corpus. En ambos casos, la modulación, trabajada desde el marco de la GSF, presenta la ventaja de sistematizar y conferir especificidad terminológica a la zona semántica de la obligación, tradicionalmente llamada "modalidad deóntica del enunciado", y a la zona semántica de la modalidad, en general. En los términos recién expuestos, este estudio se constituye en una mirada sobre una zona de la modalidad que ha sido poco explorada, pues la mayoría de los trabajos en este ámbito se focalizan en la valoración positiva o negativa (i.e. polaridad), de mayor o menor certeza (i.e. modalización), que el hablante hace de la información.

Específicamente, en este trabajo, nos preguntamos si en el manual, en general, y en los manuales de cada área disciplinar, en particular, se utilizan realizaciones más o menos moduladas, congruentes o gramaticalmente metafóricas. Dado que las realizaciones más congruentes se relacionan con un modo simple, directo y ontogenético de realización del significado e inversamente, las más moduladas (o gramaticalmente metafóricas) con uno más complejo y abstracto, los resultados de este estudio nos permitirán visualizar qué manuales presentan el modo más simple para regular la inserción disciplinar y cuáles el más abstracto; al mismo tiempo que potencia un acercamiento al aspecto interpersonal de la metáfora gramatical, en un contexto en el que suele estudiarse el grado de informatividad, usándose para ello la metáfora ideacional o nominalización (Cademártori, Parodi \& Venegas, 2007; Ferrari, 2007; Marinkovich, 2007). 
Para desarrollar la descripción y caracterización del manual, observamos el comportamiento probabilístico de las configuraciones lexicogramaticales que, en español, realizan modulación de la cláusula, en los manuales del corpus académico PUCV-2006, vistos como una totalidad (i.e. como un género discursivo), en el marco de cada una de las cuatro áreas disciplinares formantes del corpus, así como en el de la clásica oposición entre Ciencias Sociales y Humanas y Ciencias Básicas y de la Ingeniería.

\section{Fundamentos teóricos: propiedades específicas de la lengua como sistema semiótico}

Uno de los principios fundantes de la Lingüística Sistémico Funcional (LSF) es la comprensión de la lengua como sistema semiótico. Este es un supuesto tomado del estructuralismo lingüístico, a partir del cual se comprende que la lengua es un sistema de signos cuyo fin es el ser utilizado para crear significados con el propósito de intercambiarlos. Como todo sistema semiótico, el de la lengua, se organiza en torno a dos estratos o niveles: el del contenido y el de la expresión de dicho contenido (Hjelmslev, 1974). Ambos estratos se vinculan entre sí a través de un proceso denominado "realización". Este proceso puede describirse del siguiente modo: cada estrato constituye un potencial de opciones que el hablante tiene a su disposición para significar; las elecciones realizadas por el hablante en un estrato acotan y orientan las elecciones que pueden hacerse en el estrato inmediatamente inferior. Lo común es que a una elección de contenido le corresponda una -y solo una- realización en el estrato de la expresión, así, usando un ejemplo bastante común, el sistema semiótico del semáforo presenta tres opciones a nivel de contenido: "deténgase", "pase" y "tenga precaución". Para cada una de ellas existe una realización a nivel de la expresión: color rojo, verde y amarillo, respectivamente. La relación entre contenido y expresión es de uno a uno, de modo que no es posible combinar los colores para producir otros significados. En esta idea, la lengua se diferencia del común de los sistemas semióticos en dos sentidos: respecto de su organización estratal y respecto de la construcción de significados.

Respecto de la organización estratal, como podemos apreciar en la Figura 1, el sistema de la lengua presenta una organización que no es biestratal como la del común de los sistemas semióticos, con un plano del contenido y uno de la expresión, sino que una triestratal (Caffarel, Martin \& Matthiessen, 2004), por cuanto el nivel del contenido se subdivide en otros dos niveles: el semántico y el lexicogramatical (Caffarel et al., 2004; Halliday \& Matthiessen, 2004). 


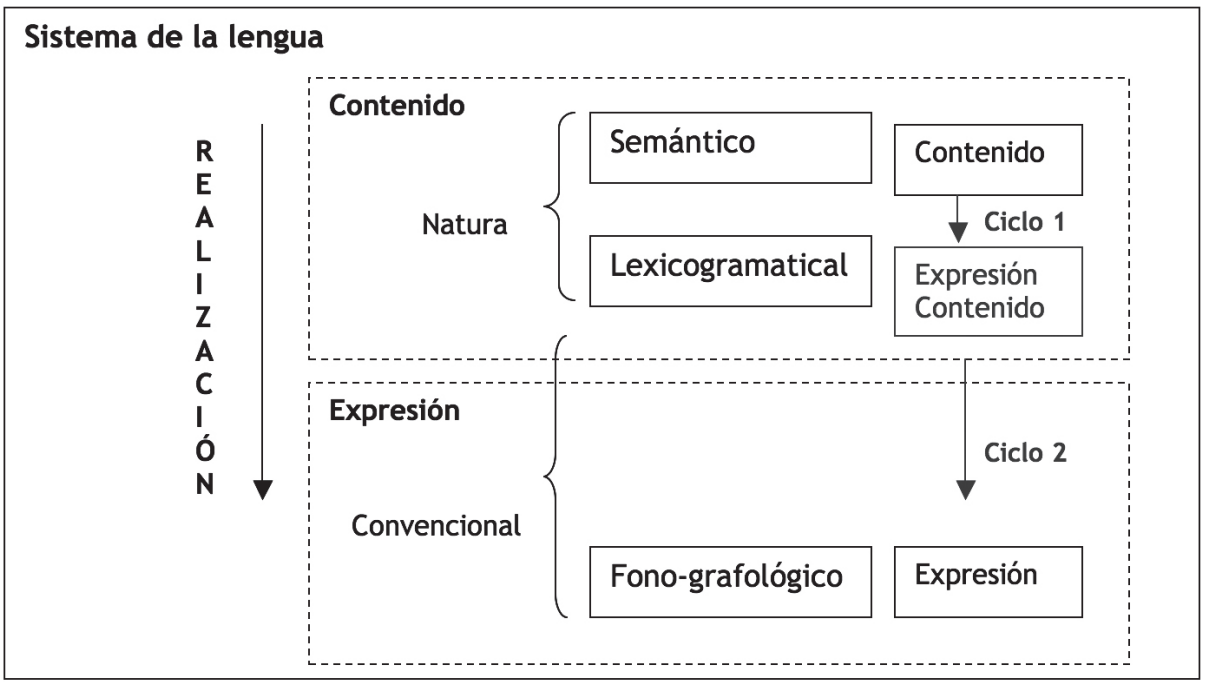

Figura 1. Relaciones interestratales al interior del sistema semiótico de la lengua.

Una de las principales implicancias de la triestratificación es que, aun cuando el estrato lexicogramatical es parte del plano del contenido, opera tanto a nivel de contenido como a nivel de expresión, en el marco de los dos ciclos realizacionales que se producen a efecto de la interfaz lexicogramatical (ver Figura 1). De este modo el lexicogramatical es contenido para la expresión fonografológica y expresión para el contenido semántico.

En términos de la construcción de significados, la lengua difiere de los otros sistemas semióticos, pues al poseer componentes funcionales, esto es, funciones complementarias y simultáneas -y no complementarias, pero excluyentes- permite significar más de una cosa al mismo tiempo (Caffarel et al., 2004). La LSF reconoce tres componentes funcionales: ideacional, interpersonal y textual. Se trata de funciones que la lengua satisface en contexto y que, dada la perspectiva contextualista de esta teoría, le confieren forma al sistema de la lengua. En otras palabras, los componentes funcionales pasan a ser metafuncionales y, como tales, son constructos teóricamente definidos como comunes a todas las lenguas (i.e. universales).

A nivel lexicogramatical, las metafunciones se realizan en la estructura funcional de la cláusula. En el marco de la GSF, la cláusula es la unidad mayor del estrato lexicogramatical, es decir, la unidad gramatical en la que integran las unidades menores (i.e. morfema, palabra y grupo o frase) para significar. Su definición es análoga a la de oración, sin embargo, esta última solo 
presenta una estructura sintagmática (i.e. compuesta de unidades, clases y subclases); mientras que la cláusula ostenta, a la vez que estructura sintagmática, una estructura funcional, conformada por funciones, postuladas, al igual que los componentes semánticos que realizan, como universales. De acuerdo a la estructura sintagmática, una cláusula puede ser finita o no-finita, es decir, puede o no contar con un verbo -o grupo verbal- que le proporcione finitud temporal y modal (i.e. 'verbo conjugado'). Respecto de la estructura funcional, en la cláusula se presenta tres configuraciones distintas y simultáneas, en consonancia y correspondencia con los tres componentes del estrato semántico. Estas estructuras son la transitiva; la interpersonal y la textual, realizando la metafunción ideacional, interpersonal y textual, respectivamente (Halliday, 1982; Matthiessen, 1995; Halliday \& Matthiessen, 2004). La Figura 2 grafica la composición estructural de las funciones.

\begin{tabular}{|c|c|c|c|c|c|}
\hline Estructura funcional & \multicolumn{5}{|c|}{ Funciones } \\
\hline Transitividad & Actor & Proceso & Meta & Circuns & ncia \\
\hline \multirow{2}{*}{ Interpersonal } & \multicolumn{2}{|c|}{ Núcleo modal } & \multicolumn{3}{|c|}{ Residuo } \\
\hline & Sujeto & Finito & Predicador & Complemento & Adjunto \\
\hline Textual & \multicolumn{2}{|c|}{ Tema } & \multicolumn{3}{|c|}{ Rema } \\
\hline
\end{tabular}

Figura 2. Composición estructural de las funciones.

El carácter bifásico de la estructura está también relacionado con la triestratificación del sistema de la lengua, de modo que la estructura funcional es, en el estrato lexicogramatical, la expresión del contenido semántico y la estructura sintagmática, el contenido para la expresión fonografológica. Ambas estructuras se vinculan entre sí por realización, así, decimos que las funciones de la estructura funcional "se realizan" en la estructura sintagmática y que una misma estructura sintagmática está realizando simultáneamente las tres estructuras funcionales.

En este nivel, la realización puede ser clasificada como más lexicalizada o más gramaticalizada y como más o menos congruente. El primer criterio de clasificación opera sobre la naturaleza de la unidad gramatical que está realizando alguna de las funciones, por ejemplo, en español, la función de núcleo modal (de la estructura interpersonal de la cláusula) se realiza en una unidad altamente gramaticalizada: los morfemas flexivos del verbo. El segundo criterio se funda en establecer si la categoría o clase gramatical es o no la que aparece en la protolengua, esto es, la que aparece en la primera fase del proceso de adquisición de una lengua. Si la categoría grama- 
tical que está realizando alguna de las funciones es la empleada en la protolengua, clasificamos la realización como "congruente"; en cambio, si se trata de otra configuración, decimos que la realización es metafórica, más precisamente, una metáfora gramatical.

Entonces, como podemos notar, la metáfora gramatical es un fenómeno que se produce en el marco de la relación de realización entre la estructura funcional y la sintagmática. Básicamente, consiste en realizar alguna de las funciones de alguna de las estructuras funcionales de la cláusula a través de una categoría gramatical diferente a la que es prototípicamente usada en la protolengua (i.e. congruente). Así, por ejemplo, para la función de "proceso" (en la estructura transitiva de la cláusula) la realización congruente es la categoría gramatical "verbo" (sea palabra o grupo verbal). Esto quiere decir que el verbo es el recurso lexicogramatical que utilizamos para representar los eventos del mundo cuando estamos aprendiendo nuestra lengua materna. Sin embargo, cuando nos hemos familiarizado con los recursos lexicogramaticales y, paralelamente, nuestra representación del mundo se hace más selecta, nosotros podemos necesitar complejizar dicha realización, por ejemplo, en vez de decir, "Colón descubrió América en el año 1492", decimos, "El descubrimiento de América fue en el año 1492", utilizando ya no un verbo -con sus respectivos participantes asociados-, sino que un grupo nominal, esto es, utilizando una categoría gramatical metafórica. Al igual que la metáfora léxica, la gramatical, constituye un recurso que expande el potencial de significar de una lengua cuando necesitamos y podemos expandirlo. Esto es importante para este estudio, pues el sistema que nos ocupa, la modulación, presenta una gradación que se sustenta en el principio de la metáfora gramatical.

\subsection{El sistema de la modulación obligativa}

En efecto, el sistema de modulación obligativa, entendido como el que aglutina las opciones semánticas y lexicogramaticales que utilizamos para realizar un requerimiento, presenta una organización sistémica basada en los grados de obligación implicados en una demanda. Desde el estrato semántico, se reconocen, al menos, tres grados de modulación obligativa: alto, medio y bajo (Martin, 1992; Halliday \& Matthiessen, 2004). El grado alto corresponde al mayor grado de obligación, esto es, a lo más directo o explícitamente apelativo. A medida que el grado de obligación disminuye, aumenta el grado de modulación implicado en la demanda. En consecuencia, más modulado quiere decir que la demanda se presenta de un modo menos directo.

Ahora bien, asignar un grado de obligación depende de dos aspectos contextuales. De una parte, depende de cuál es la posición jerárquica que el hablante ostenta respecto del destinatario de su demanda y, de otra, del compromiso entre el "tú" del diálogo y lo que es demandado (Martin et al., 1997; Halliday \& Matthiessen, 2004). La preferencia por la atenuación o metaforización de la demanda, entonces, puede ser entendida como un mecanismo de cortesía, al relacionarse con el vínculo formal que se establece entre los participantes del evento de habla (por ejemplo, 
de experto a experto, en el ámbito que nos ocupa); o como una estrategia funcional, al estar relacionado con los mecanismos más explícitos que se requiere usar cuando tales participantes no están co-presentes y, por tanto, la intención comunicativa no puede apoyarse en factores tales como la entonación (o modulación, según terminología del Círculo de Praga) y, entonces deben "modularse", para hacer explícito el significado.

A nivel lexicogramatical, esta gradación se corresponde con la congruencia de la realización, de modo que la configuración que realiza obligación del modo más directo o menos modulado es también la más congruente y simple, desde el punto de vista ontogenético (Matthiessen, 1995); en tanto que la que realiza un grado bajo de obligación, esto es, la más modulada, es la más metafórica.

Si bien menos directas y, en consecuencia, más abiertas a la negociación, las configuraciones más moduladas o metafóricas se caracterizan, a nivel lexicogramatical, por ser más complejas y abstractas. Su uso está asociado a registros escritos que ostentan un alto grado de especialización (Gutiérrez, 2007) y se coligan, como toda metáfora, con un estadio avanzado en el proceso de adquisición de una lengua (Halliday, 1982, 1984; Matthiessen, 1995). Inversamente, se considera que las realizaciones lexicogramaticales menos moduladas son las que mayor congruencia tienen con el significado (que está siendo realizado), las que están presentes en el habla infantil (o protolengua) (Matthiessen, 1995) y, en consecuencia, las más simples y directas.

En español, el sistema de la modulación obligativa presenta seis opciones (Gutiérrez, 2007), dos para cada uno de los grados -alto, medio y bajo- de la obligación, definidos desde el estrato semántico, tal como puede observarse en la Figura 3. 


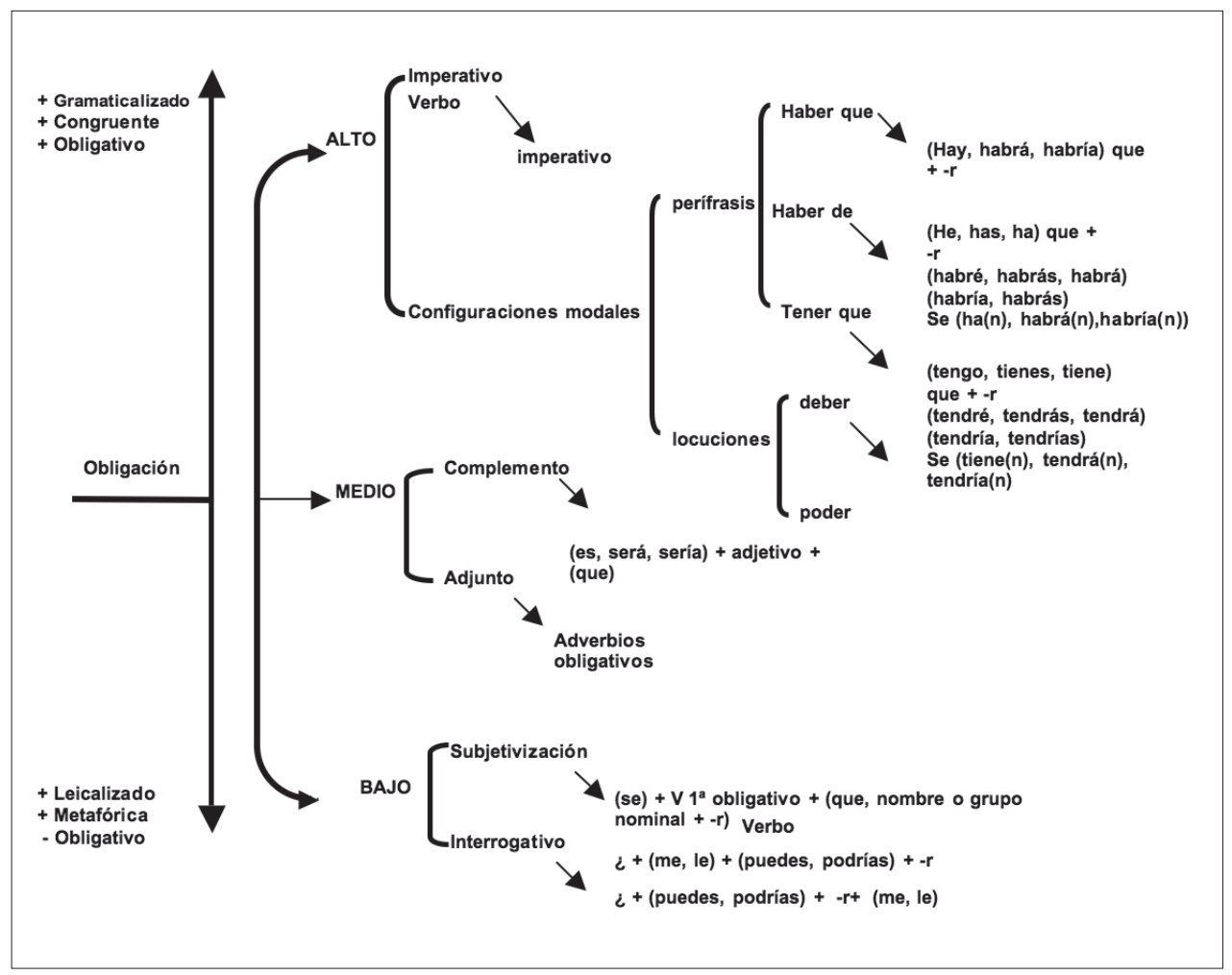

Figura 3. Gradiente de la obligación para el español.

Como ya se explicitó y como notamos en la Figura 3, las configuraciones están dispuestas en torno a los tres movimientos que modulan la obligación implicada en una demanda. Esta gradación tiene un comportamiento fractal, lo que quiere decir que se replica al interior de cada uno de los términos del sistema, tal como se presenta en la Figura 4. 
En español, además, se presenta una relación directamente proporcional entre grado de obligatoriedad del requerimiento, congruencia, gramaticalización/lexicalización y la posición en la estructura funcional de la cláusula como intercambio (Gutiérrez, 2007). Así, una configuración cuya posición en la estructura funcional sea la núcleo modal (i.e. SUJETO + FINITO) o núcleo de la negociabilidad, es más gramaticalizada, más congruente y, en consecuencia, más directamente obligativas.

a) Grado alto de obligación: imperativo y configuraciones modales obligativas: en el contexto proporcionado por el grado alto, el 'imperativo' es la realización más congruente y, en este sentido, la que realiza con mayor fuerza o menor modulación la obligación implicada en una demanda. Se trata de una configuración altamente gramaticalizada cuya realización corresponde al núcleo modal congruente de una demanda, es decir, a un sujeto de segunda persona y a un finito temporal de presente del imperativo, por ejemplo, "mire" en "mire detenidamente la figura" (ejemplo tomado del corpus PUCV-2006 AC-QUI-ma408).

Las "configuraciones modales obligativas" comienzan a distanciarse de la congruencia realizada por el imperativo. Esto pues, aun cuando desempeñan la función de núcleo modal y pueden realizarse con sujeto de segunda persona y en tiempo presente, no admiten la flexión en modo imperativo, dada la naturaleza léxica de los verbos que funcionan como operadores modales y finito (Hernández, 1995).

b) Grado medio de la obligación: adjetivos y adverbios obligativos: el grado medio de la obligación, por su parte, agrupa a configuraciones lexicogramaticales que realizan el significado obligativo fuera del marco del núcleo modal. Es decir, la realización lexicogramatical se ha desplazado hacia alguna de las otras funciones de la cláusula como intercambio, específicamente el desplazamiento es hacia la función de complemento, en el caso del adjetivo o hacia la de adjunto, en el caso del adverbio. Entre ellas, la que presenta mayor congruencia $y$, por tanto, mayor intensidad del significado es la realización en la función de complemento, pues el complemento tiene la potencialidad de ser sujeto y, en consecuencia, de ser parte del núcleo modal (Martin et al., 1997). El adjunto, en cambio, y al menos en el caso de la modulación, tiende a reforzar lo que ya está realizado en el núcleo modal, es decir, la cláusula ya está modulada, por ejemplo, "necesariamente" en "deben ser los valores necesariamente idénticos" (AC-QUI-ma418), solo refuerza la obligación implicada en "deben".

En concordancia con el hecho de que nos encontramos frente a realizaciones que se distancian del núcleo modal, función que en español está altamente gramaticalizada, las configuraciones del grado medio son configuraciones que transportan léxicamente la obligación, sometiéndose a la apertura y variabilidad que caracteriza al paradigma lexical (Bosque \& Demonte, 1999; Halliday, 2002a, 2002b). 
c) Grado bajo: verbos obligativos y modo interrogativo: las configuraciones de grado bajo son las realizaciones que menor congruencia mantienen con el núcleo modal de la cláusula imperativa. De una parte, la conformada por 'verbo obligativo + (que)' no admite un sujeto de segunda persona, pues en tal caso se implica la inclinación más que la obligación (Gutiérrez, 2007); tampoco puede ser flexionada en modo imperativo, pues la naturaleza léxica de los verbos lo impide (Hernández, 1986; Porto, 1991; Di Tullio, 1997). De otra, la configuración correspondiente a cláusulas interrogativas es la realización de menor congruencia, pues en ella se implica un cambio de modo clausular, específicamente, del imperativo al interrogativo, construyendo lo que llamamos comúnmente una "orden indirecta".

\section{La investigación}

\subsection{Objetivos}

El cuestionamiento que orienta este estudio es ¿cuál es el grado de obligación que con mayor probabilidad se utiliza en el género manual? Esta pregunta se operacionaliza en el objetivo general de investigación, el cual es caracterizar el género manual, a partir de la variación que presenta el perfil probabilístico de las configuraciones lexicogramaticales que, en español, realizan modulación obligativa de la cláusula.

\subsection{Variables}

En este estudio se trabaja con variables descriptivas categóricas y cuantitativas, ya que las categóricas han sido definidas en el marco de la exposición del modelo de análisis y de sus fundamentos teóricos, en este apartado solo presentamos su operacionalización.

a) Configuraciones lexicogramaticales que realizan modulación de obligación de la cláusula (variable categórica): Las configuraciones lexicogramaticales que realizan modulación de obligación, conforman, tal como lo presentáramos en apartados anteriores, una red sistémica, donde cada término corresponde a una variable. La Tabla 1 presenta dichas variables, distribuyéndolas por grado de intensidad. Paralelamente, da cuenta de la sintaxis empleada para desarrollar las búsquedas automáticas en El Grial.

Respecto de la Tabla 1, necesitamos hacer dos precisiones que tienen relación con el grado de detalle (o delicacy) al que aspiramos en este estudio:

(i) En primer lugar, todas las configuraciones que tienen a un verbo o a un grupo verbal como una de sus unidades formantes son buscadas en la interfaz de consulta El Grial (www.elgrial. $\mathrm{cl}$ ), conjugando distintos sujetos y finitos. Esto, pues reconocemos, como quedó explicado en el marco teórico, que se produce una gradación de la obligación en la interacción con los 
sistemas de tiempo y persona (i.e. con la flexión del núcleo modal). Desde esta perspectiva, asumimos que, en general, el único finito temporomodal que queda excluido de la realización de obligación es el pasado. En efecto, como concuerdan en reconocer Rojo y Veiga (1999), Halliday y Matthiessen (2004), Palmer (2001) y Klinge y Hoeg (2005), el pasado en combinación con cualquier sujeto no realiza obligación (ni inclinación), pues no constituye propuestas, sino que proposiciones.

La gradación temporal y de persona que presenta el sistema de obligación excede el nivel de detalle que se pretende en esta descripción, razón por la cual, para cada una de las configuraciones que presentan esta gradación se han contabilizado todas las opciones presentadas en la Tabla 1, pero considerándolas en su conjunto.

(ii) En segundo lugar, algunas de las configuraciones obligativas están sometidas a la variabilidad del paradigma lexical. Tal variabilidad, al igual que la de tiempo y persona, excede el grado de detalle de este trabajo, por lo cual nuestro esfuerzo se limita a realizar búsquedas homogéneas, para todos los registros del corpus, siguiendo la propuesta desarrollada por Gutiérrez (2007) para este sistema.

En relación a este mismo tipo de configuración, es necesario señalar que el significado léxico también puede ser graduado en términos de más o menos obligativo. Sin embargo, este es un asunto propio de estudios lexicográficos o evaluativos (i.e. appraisal), motivo por el cual, las opciones son contabilizadas sin pasar al detalle de la gradación, sino que focalizándonos en un nivel más grueso, que es más cercano a los patrones lexicogramaticales.

b) Frecuencia absoluta de instanciación de las configuraciones lexicogramaticales (variable cuantitativa): medida de estadística descriptiva cuyo valor es un número que representa la cantidad de veces que una configuración es instanciada en el marco de un registro en particular. El etiquetador de corpus que emplearemos entrega directamente los resultados de las búsquedas por frecuencia.

c) Probabilidad de instanciación (variable cuantitativa): medida de estadística descriptiva que permite predecir cuán probable es que, dadas las mismas condiciones, un suceso pueda volver a ocurrir. Dado que trabajamos sobre la base de una representación sistémica, en la cual el término de un sistema puede ser la condición de entrada a otro sistema menor, el cálculo de la probabilidad utilizado es el de probabilidades condicionadas. Las probabilidades condicionadas permiten calcular la probabilidad de ocurrencia en el marco de una condición o contexto mayor, por ejemplo, el cálculo de la probabilidad de ocurrencia del imperativo está condicionado al contexto mayor representado por el grado alto de la obligación. El cálculo de la probabilidad se desarrolla sobre el de frecuencia absoluta y se presenta en términos porcentuales. Para determinar si la variación entre las probabilidades de ocurrencia es estadísticamente significativa se 
Tabla 1. Configuraciones lexicogramaticales que realizan obligación.

\begin{tabular}{|c|c|c|}
\hline & \begin{tabular}{|l|} 
Configuración \\
lexicogramatical
\end{tabular} & Forma de búsqueda \\
\hline \multirow[t]{4}{*}{$\begin{array}{l}\text { GRADO } \\
\text { ALTO }\end{array}$} & $\begin{array}{l}\text { Verbo en modo } \\
\text { imperativo }\end{array}$ & $\begin{array}{l}\text { Búsqueda } 1 \text { (simple): } \\
\text { Token 1.1: POS= V IMP }\end{array}$ \\
\hline & \multirow[t]{3}{*}{$\begin{array}{l}\text { Verbo modal de } \\
\text { obligación }\end{array}$} & $\begin{array}{l}\text { Búsqueda } 2 \text { (simple): } \\
\text { Token 2.1: Forma \{ Debo, debemos, debes, debe, deben, deberé, deberemos, } \\
\text { deberás, deberá, deberán, debería, deberíamos, deberías, deberían, debiese, } \\
\text { debiésemos, debieses, debiesen }\end{array}$ \\
\hline & & $\begin{array}{l}\text { Búsqueda } 3 \text { (compleja): } \\
\text { Token 3.1: Forma \{ Hay, habría, habrá, tengo, tenemos, tienes, tiene, tienen, } \\
\text { tendré, tendremos, tendrás, tendrá, tendrán, tendría, tendríamos, tendrías, } \\
\text { tendrían } \\
\text { Token 3.2: Forma = que } \\
\text { Token 3.3: POS= VDB INF }\end{array}$ \\
\hline & & $\begin{array}{l}\text { Búsqueda } 4 \text { (compleja): } \\
\text { Token 4.1: Forma }\{\text { He, hemos, has, ha, han, habré, habremos, habrás, habrá, } \\
\text { habrán, habría, habríamos, habrías, habrían } \\
\text { Token 4.2: FORMA = de }\end{array}$ \\
\hline \multirow[t]{2}{*}{$\begin{array}{l}\text { GRADO } \\
\text { MEDIO }\end{array}$} & $\begin{array}{l}\text { Ser + adjetivo } \\
\text { obligación }\end{array}$ & $\begin{array}{l}\text { Búsqueda } 5 \text { (compleja): } \\
\text { Token 5.1: Forma \{ Es, será, sería } \\
\text { Token 5.2: Forma \{ Necesario, obligatorio, aconsejable, permitido, } \\
\text { recomendable, }\end{array}$ \\
\hline & $\begin{array}{l}\text { Adverbios de } \\
\text { obligación }\end{array}$ & $\begin{array}{l}\text { Búsqueda } 6 \text { (simple): } \\
\text { Token 6.1: Forma \{ Necesariamente, obligatoriamente, debidamente, } \\
\text { indebidamente, apropiadamente, rigurosamente, obligadamente, } \\
\text { fundamentalmente, adecuadamente, forzosamente, forzadamente }\end{array}$ \\
\hline \multirow[t]{2}{*}{$\begin{array}{l}\text { GRADO } \\
\text { BAJO }\end{array}$} & Verbo Obligativo & $\begin{array}{l}\text { Búsqueda } 7 \text { (compleja): } \\
\text { Token 7.1: Forma = se } \\
\text { Token 7.2: Forma \{ Sugiere, sugieren, autoriza, autorizan, necesita, } \\
\text { necesitan, requiere, requieren, permite, permiten, aconseja, aconsejamos, } \\
\text { recomienda, recomiendan, permite, permiten, aconseja, aconsejan, } \\
\text { recomienda, recomiendan, da, dan, exige, exigen, obliga, obligan, pide, } \\
\text { piden, ruega, rogan, solicita, solicitan } \\
\text { Búsqueda } 8 \text { (simple): } \\
\text { Token 8.1: Forma \{ Sugiero, sugerimos, autorizo, autorizamos, necesito, } \\
\text { necesitamos, requiero, requerimos, permito, permitimos, aconsejo, } \\
\text { aconsejamos, recomiendo, recomendamos, exigo, exigimos, obligo, obligamos, } \\
\text { pido, pedimos ruego, rogamos, solicito, solicitamos }\end{array}$ \\
\hline & Interrogativos & $\begin{array}{l}\text { Búsqueda } 9 \text { (compleja): } \\
\text { Token 9.1: FORMA }=i \\
\text { Token 9.2: FORMA }\{\text { Puedes, podrías } \\
\text { Token 9.3: POS = V } \\
\text { Modo y tiempo: VDB INF }\end{array}$ \\
\hline
\end{tabular}


utiliza la comparación de proporciones Z (normal estandarizada), estimándose un error global del $3 \%$.

\subsection{El corpus: caracterización y explotación}

El corpus de este estudio está conformado, como ya se ha dicho, por los manuales del corpus académico PUCV-2006. Cuantitativamente, se trata de un corpus conformado por 23.489.036 palabras que corresponden a 126 textos distribuidos por disciplina, como se muestra en la Tabla 2.

Tabla 2. Distribución del corpus de trabajo por disciplina.

\begin{tabular}{|l|c|r|}
\hline & Manuales & \multicolumn{1}{|c|}{ Palabras } \\
\hline Psicología & 31 & 4.925 .931 \\
\hline Trabajo Social & 15 & 2.465 .747 \\
\hline Química Industrial & 31 & 9.161 .146 \\
\hline Ingeniería en Construcción & 49 & 6.936 .212 \\
\hline Total & 126 & 23.489 .036 \\
\hline
\end{tabular}

Para efectos de este estudio, el corpus se cuantifica respecto de cláusulas, pues el sistema de la obligación modula sobre esta unidad y no sobre la palabra, que es la unidad típicamente usada para la cuantificación de un corpus.

Las cláusulas que contabilizamos son las finitas, arriba definidas, pues estas son las que realizan la estructura funcional de la cláusula como intercambio, sobre la que opera el sistema de la modulación. Desde esta perspectiva, la cuantificación del corpus es la que se presenta en la Tabla 3.

Tabla 3. Distribución de las cláusulas en los registros del corpus.

\begin{tabular}{|l|r|}
\hline Registro & Total de cláusulas \\
\hline Psicología & 415.497 \\
\hline Trabajo Social & 215.387 \\
\hline Química Industrial & 720.755 \\
\hline Ingeniería en Construcción & 524.660 \\
\hline Corpus total & 1.876 .299 \\
\hline
\end{tabular}

Ahora bien, los manuales serán analizados en tres niveles. De una parte, se observa la totalidad del corpus, esto es, como se señaló, el manual como género discursivo, sin hacer distinción de área disciplinar. De otra, se compararán los manuales respecto de cada una de las áreas en 
particular, así como respecto de la agrupación Ciencias Sociales y Humanas (i.e. Psicología y Trabajo Social) y Ciencias Básicas y de la Ingeniería (i.e. Ingeniería en Construcción y Química Industrial). Estos tres niveles de análisis pueden visualizarse en la Figura 4.

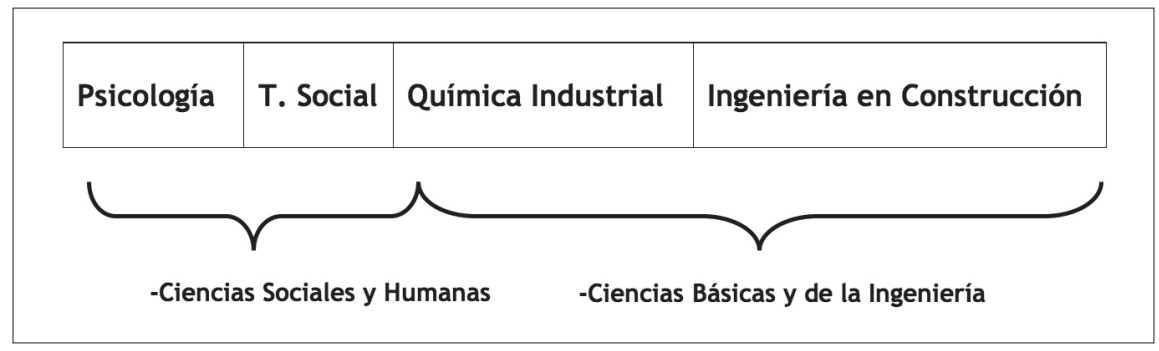

Figura 4. Distribución de los manuales por área disciplinar.

De cada una de las configuraciones de la red sistémica de la obligación, observamos la frecuencia absoluta de ocurrencia en los registros del corpus. Para extraer este dato se utiliza el etiquetador morfosintáctico El Grial (www.elgrial.cl), empleándose como secuencia de búsquedas las estructuras sintagmáticas de cada configuración (que son los que aparecen en la Tabla 1).

Sobre el dato de la frecuencia absoluta, calculamos la frecuencia relativa de ocurrencia o probabilidad. Para ello, utilizamos el estadístico de probabilidades condicionadas (en este caso, condicionadas a cada grado y cada grado condicionado a los otros dos), calculadas.

\section{Resultados}

A continuación, se presentan los resultados siguiendo la organización de la red sistémica de la obligación. De este modo, se consigue mostrar el comportamiento probabilístico de las variables observadas, a partir del contraste al interior de cada uno de los grados que realizan obligación, así como del contraste entre grados.

\subsection{Grado alto: imperativo v/s configuraciones modales}

Observemos el Gráfico 1. 


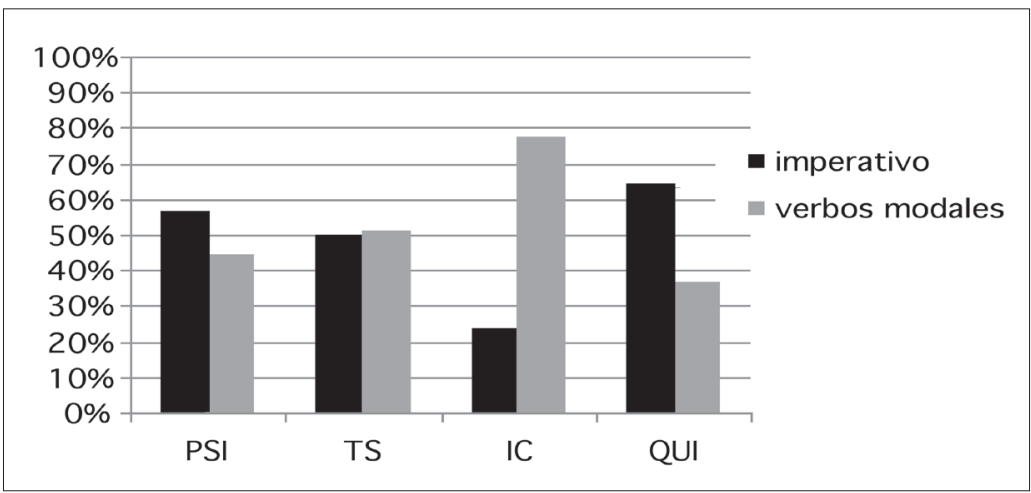

Gráfico 1. Comparación de los perfiles de ocurrencia de las configuraciones de grado alto.

Lo primero que podemos notar, a partir de la distribución de los datos en el Gráfico 1, es que cada una de las configuraciones de grado alto, presenta su particular modo de ocurrir en el marco de cada una de las áreas disciplinares. Así, en Psicología (PSI) y Química (QUI) se prefiere utilizar el imperativo; mientras que en Ingeniería Industrial (IC) se prefieren utilizar las configuraciones modales y en Trabajo Social (TS) se utilizan las dos con la misma probabilidad de ocurrencia.

La descripción del Gráfico 1 configura un panorama bastante variable, sin embargo, podemos inducir otro escenario a partir de la observación de la agrupación Ciencias Sociales y Humanas, de una parte, y Ciencias Básicas y de la Ingeniería, de otra. Desde aquí, notamos que las configuraciones de grado alto tienden a coexistir y a estrechar la probabilidad de su ocurrencia en el marco de las Ciencias Sociales y Humanas (i.e. Psicología y Trabajo Social), tal como lo muestra el Gráfico 2, creemos que esta coexistencia de las configuraciones se relaciona con las diferentes movidas retóricas de los manuales de estas áreas (Parodi, 2008), así, por ejemplo, el imperativo suele aparecer en el marco de las instrucciones para ejercitar; en cambio, las configuraciones con verbos modales se asocian a las definiciones de conceptos que aparecen en los manuales. Inversamente, en el marco de las Ciencias Básicas y de la Ingeniería, la tendencia es a distanciar dichas probabilidades, sea a favor del 'imperativo', como en Química Industrial, o de los 'verbos modales obligativos', como en Ingeniería en Construcción.

Al comparar la ocurrencia de las dos configuraciones del grado alto en el marco de la agrupación Ciencias Sociales y Humanas y Ciencias Básicas y de la Ingeniería, el fenómeno de la coexistencia persiste. 


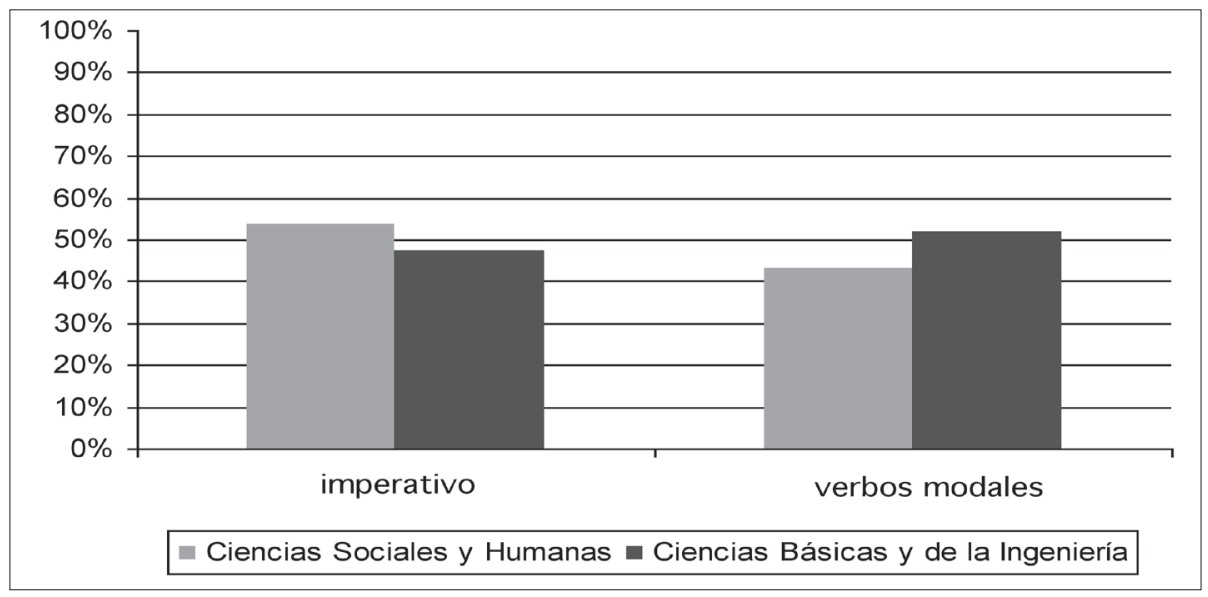

Gráfico 2. Probabilidad de ocurrencia de las configuraciones de grado alto en el contexto de la oposición Ciencias Sociales y Humanas - Ciencias Básicas y de la Ingeniería.

La prueba estadística aplicada a estos resultados no mostró diferencias significativas entre las ocurrencias, de modo que es posible afirmar que la coexistencia de ambas configuraciones caracteriza al género manual y que esta forma de construir la obligación no varía en el contexto de la variable de campo 'área disciplinar'.

Otros estudios en esta misma línea han evidenciado que la mayor probabilidad de uso del imperativo se presenta en registros de interacción didáctica, en los que se pretende simplificar el acceso a un conocimiento, dado que es la forma más simple y directa (más congruente) de construir la obligación implicada en un requerimiento (Gutiérrez, 2008). Las configuraciones modales, en cambio, tienen una mayor probabilidad de ser utilizadas en registros escritos de alta especialización (Gutiérrez, 2007). A partir de estos datos, estamos en condiciones de afirmar que el manual en general, mezcla la tendencia a la didactización, manifiesta a través del imperativo, con la especialización propia de los géneros académicos universitarios, manifiesta a través del uso de configuraciones con verbos modales.

\subsection{Grado medio: ser + adjetivo obligativo adverbios}

El perfil probabilístico que puede ser inducido a partir de las ocurrencias de las configuraciones de grado medio se asemeja al de grado alto. En efecto, 'ser + adjetivo obligativo' y 'adverbios' tienden a coexistir en los manuales de las Ciencias Sociales y Humanas y a distanciarse en las Ciencias Básicas y de la Ingeniería, como puede observarse en el Gráfico 3, a continuación. 


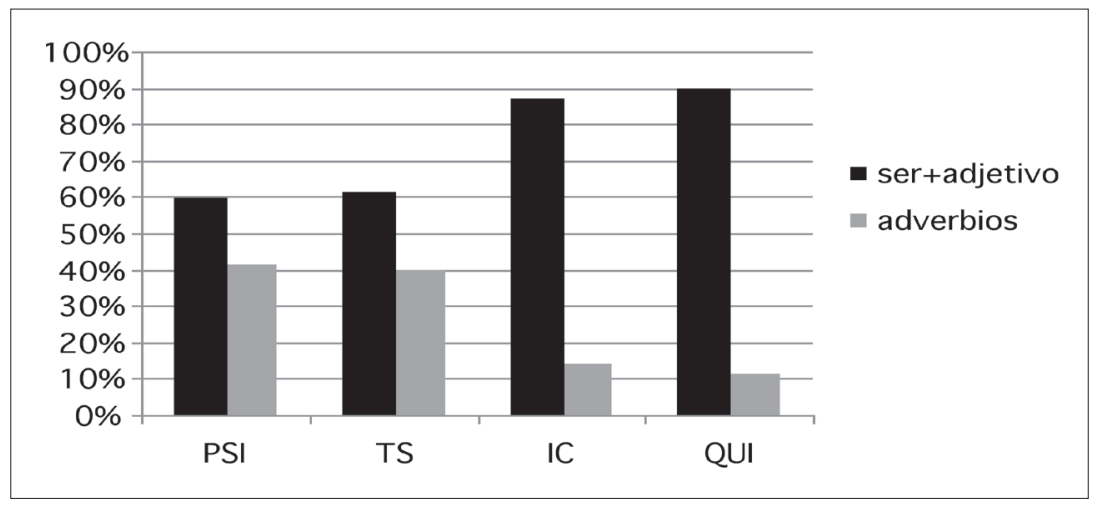

Gráfico 3. Comparación de los perfiles de ocurrencia de las configuraciones de grado medio.

La coexistencia de ambas configuraciones es una característica de un mayor grado de especialización, tal como se demuestra el estudio de Gutiérrez (2007). En consecuencia, las Ciencias Sociales y Humanas muestran una mayor tendencia hacia la especialización; en tanto que las Ciencias Básicas y de la Ingeniería tienden a comportarse como lo hacen los registros más divulgativos y, en consecuencia, son este último grupo de ciencias, las que presentan la iniciación a la disciplina de un modo mucho más didáctico.

A diferencia del grado alto, en el medio, se opta por la configuración 'ser + adjetivo obligativo' por sobre la de 'adverbio' en el manual de todas las áreas disciplinares. Las mayores ocurrencias de la configuración 'ser + adjetivo obligativo' se presentan en Química Industrial, le sigue Ingeniería en Construcción, luego Psicología y finalmente, Trabajo Social. Con esta preferencia, se marca la asimetría en la relación de los hablantes, de modo tal que experto-aprendiz no co-construyen el conocimiento, más bien, el aprendiz es instruido respecto de un marco conceptual y procedural sobre el cual, solo posteriormente, andamiará o construirá el conocimiento específico de la disciplina. También, en este caso, el predominio de la configuración 'ser + adjetivo obligativo' tiene relación con el carácter didáctico y divulgativo del manual (Gläser, 1993; Marinkovich, 2005), pues dicha configuración es ampliamente utilizada en el discurso de reformulación de la ciencia, como lo ha demostrado Rail (2007).

En otras palabras, y tal como se muestra en el Gráfico 4, son las Ciencias Básicas y de la Ingeniería, comúnmente llamadas "ciencias duras", las que regulan de modo más fuerte o, precisamente, más "duro", la inserción al conocimiento disciplinar, probablemente debido a la exactitud de los procedimientos que se deben aprender. 


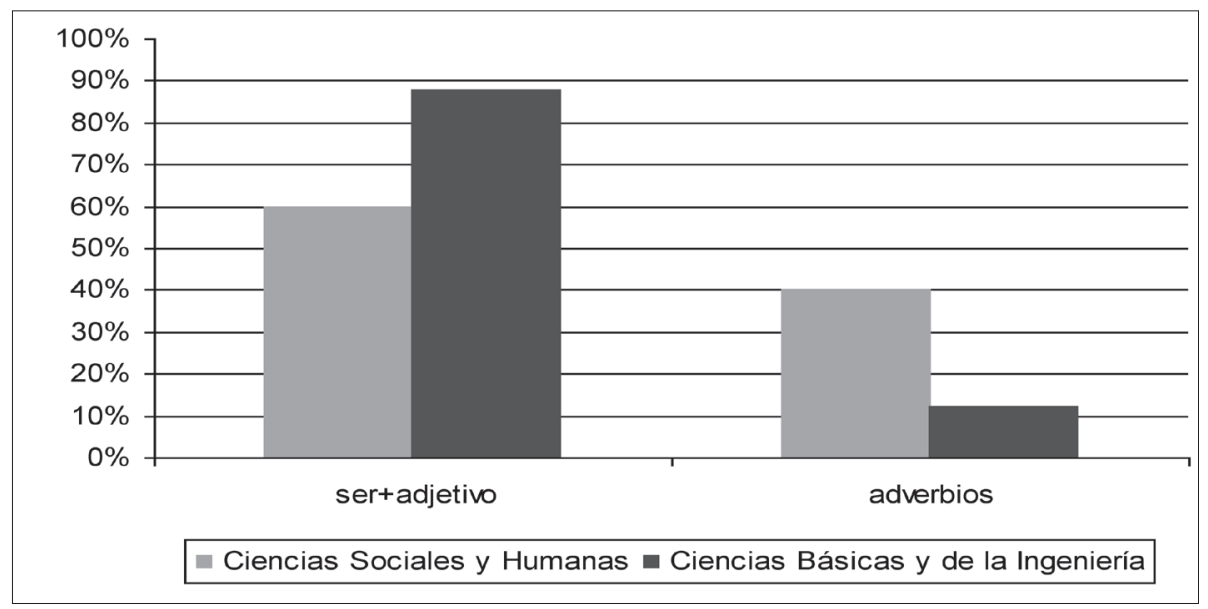

Gráfico 4. Probabilidad de ocurrencia de las configuraciones de grado medio en el contexto de la oposición Ciencias Sociales y Humanas - Ciencias Básicas y de la Ingeniería.

La forma más directa y congruente, preferida por las Ciencias Básicas y de la Ingeniería, podría ser un modo de iniciación que genere mayor seguridad en los estudiantes respecto de lo que aprenden, pues el marco conceptual y procedural se les presenta como un cuerpo de conocimiento estático, no sujeto a variación, con el cual no contamos en las Ciencias Sociales y Humanas, tal vez, porque las realidades se describen como si estuviesen sometidas a constante variación y, consecuentemente, como una zona sujeta a la co-construcción.

Las Ciencias Sociales y Humanas, por su parte, son las que, respecto del grado medio de la obligación, serían más moduladas, es decir, más abiertas a la negociación del conocimiento y más interactivas (i.e. más "blandas"), atenuándose la distancia experto-aprendiz.

\subsection{Grado bajo}

El grado bajo, por su parte, es el que sigue al grado alto en orden de la cantidad de ocurrencias que se presenta en el corpus total (tal como se muestra en el Gráfico 6).

La configuración más metafórica (i.e. metáfora de modo clausular), correspondiente a la pregunta del tipo ¿puede desarrollar la actividad?, es la que presenta la más baja probabilidad de ocurrencia en los manuales, en general, y en cada área, en particular, tal como se muestra en el Gráfico 5. 


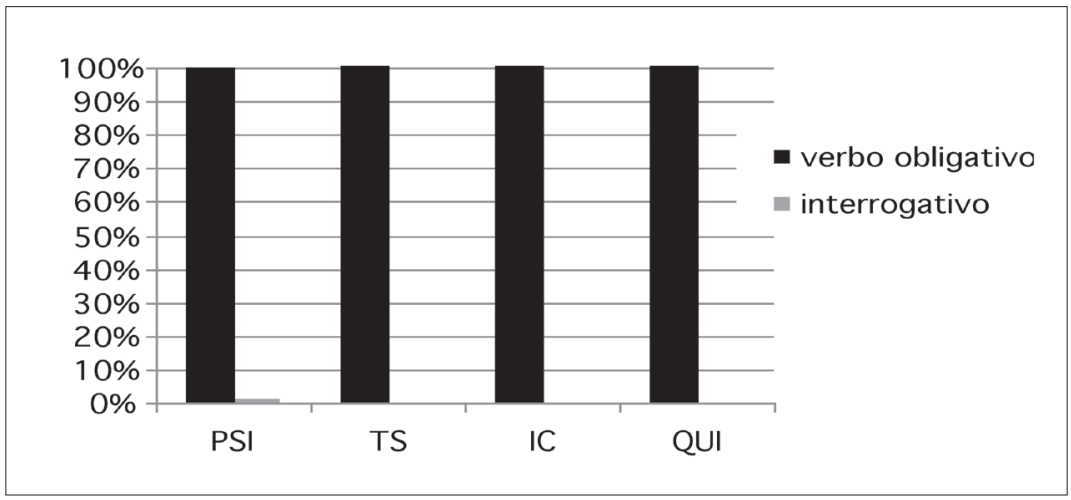

Gráfico 5. Comparación de los perfiles de ocurrencia de las configuraciones de grado bajo.

Estos resultados están en concordancia con estudios que previamente muestran que se trata de una configuración que caracteriza a la oralidad de menor especialización (Halliday, 2002c; Gutiérrez, 2007). En efecto, la configuración con interrogativo implica un alto grado de interactividad que encubre la relación experto-aprendiz tras la "orden indirecta" o petición, cuando la distancia experto-aprendiz está afecta a la variable modo (i.e. oral-escrito).

De lo anterior se desprende que la configuración con verbo obligativo, en la cual la demanda se expone como un deseo del "yo" del intercambio dialógico es la propia de los manuales. Del mismo modo que en los casos anteriores, se trata de la configuración más congruente, en el marco del grado bajo de la obligación. Su utilización hace más evidente el carácter de la regulación a través de la explicitación léxica del grado de obligación. En consecuencia, también puede ser visualizada como una configuración que complejiza el acceso al conocimiento, por cuanto la atención se centra mayoritariamente en el grado de obligación implicado, es decir, en el carácter de la negociación más que en lo que está siendo negociado.

\subsection{Comparación entre grados}

Respecto de la comparación entre grados, los resultados muestran que las configuraciones realizadoras del grado medio de la obligación son las que, en el marco del corpus total de este trabajo, presentan la menor probabilidad de ocurrencia, tal como puede observarse en el Gráfico 6. 


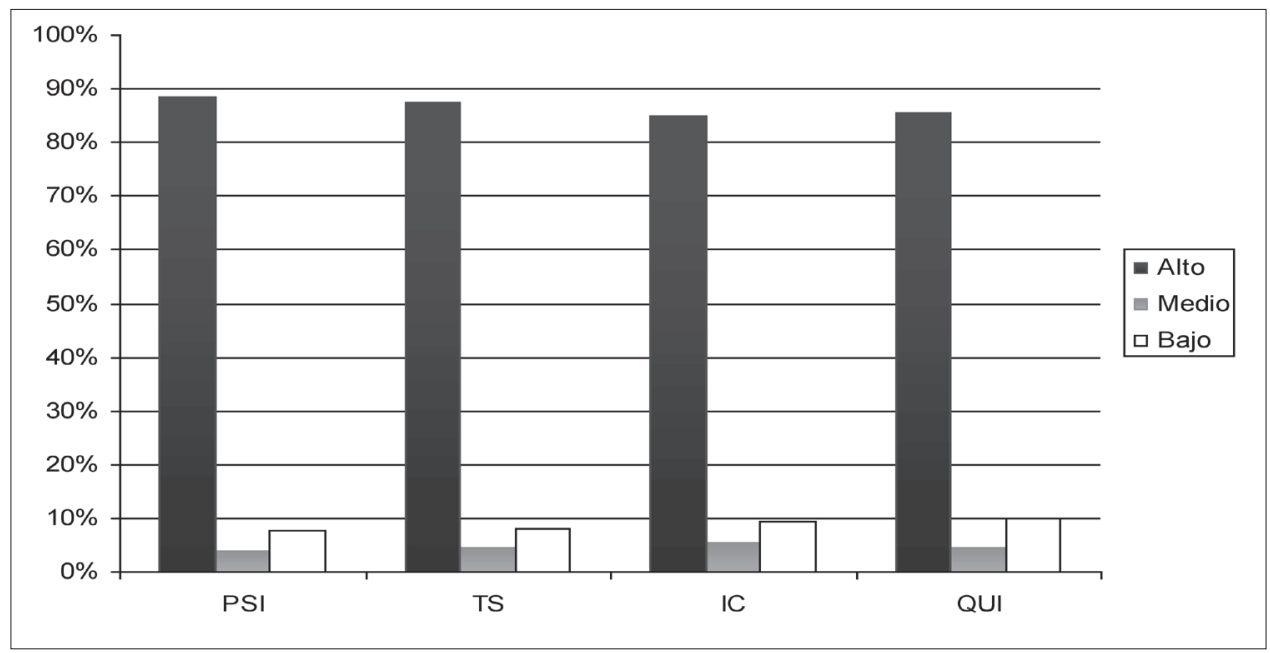

Gráfico 6. Comparación de la ocurrencia de los Grados de obligación.

Con toda seguridad, esto se debe a que ambas configuraciones son características de registros en donde el tenor de la relación entre los hablantes es de experto a experto (Gutiérrez, 2007) y no de experto a aprendiz, como en el caso del manual.

Los manuales construyen el significado de la obligación utilizando, principalmente, configuraciones pertenecientes al grado alto de obligación (ver Gráfico 6). Este resultado pone de manifiesto no solo la naturaleza regulativa asignada al género discursivo manual (Parodi et al., 2008); sino que también nos permite caracterizar dicha función como una altamente obligativa. Por supuesto, no podemos dejar de recordar que las configuraciones de grado alto son también las más directas y simples para realizar una orden y, en consecuencia, creemos que su profusa ocurrencia marca una tendencia hacia la didactización del conocimiento que es propia de los manuales (Gläser, 1993; Parodi, 2008). En efecto, el discurso didáctico busca facilitar el acceso al conocimiento, a través de la utilización de diversos recursos asociados a las realizaciones más simples y directas de una lengua (Halliday, 2004a, 2004b) como lo son el 'imperativo' y las 'configuraciones con verbo modal'. En este último sentido, la alta ocurrencia del grado alto lo iguala a los registros, que respecto del tenor, son de menor especialización (Halliday, 2004a, 2004b; Gutiérrez, 2007). Se trata, entonces, de un género que si bien es especializado respecto de su temática (i.e. variable de campo), no lo es respecto de la interrelación de los participantes, la cual es, en todos los contextos acá revisados, marcadamente asimétrica (específicamente, de experto a aprendiz). 
Ahora bien, si partimos de la idea funcionalista de que la lengua es un sistema virtual al cual le subyacen múltiples sistemas funcionales (Peronard, 1988), es decir, un polisistema (Firth, 1968), formado de tantos sistemas como funciones contextuales existan; aprender una disciplina a nivel universitario es aprender un nuevo sistema funcional, un nuevo código para intercambiar significados (Halliday, 2004b). Este aprendizaje, que posibilita el acceso ya no a una comunidad de habla, sino que a una comunidad discursiva (Ivanic, 1998; Swales, 2000), podría ser análogo al proceso de aprender la lengua materna. Así, los estudiantes que se inician en una disciplina serían como niños aprendiendo su lengua materna. En un primer momento, se necesitará controlar el ambiente. Esta es una necesidad fundamental que ocupará toda la atención del iniciado. Se trata de un estadio en el que requiere de apropiarse del léxico de la disciplina, más que de los recursos de negociación. En esta primera instancia podría no ser propicio presentar el conocimiento disciplinar como algo relativo y cambiante (i.e. como algo negociable), más bien se hace necesario proporcionar un marco firme, que sea sentido como seguro, para andamiar el dominio del ambiente disciplinar a través del léxico. En este contexto, el manual, según la caracterización que hemos presentado acá, es un género que facilita el dominio de los conceptos básicos de una determinada disciplina. Esto se manifiesta con claridad en la preferencia de las configuraciones de más directas y simples, esto es, las más congruentes para realizar lexicogramaticalmente la regulación. Estas configuraciones son también las que implican el mayor grado de obligatoriedad en la regulación, cerrando de este modo el espacio a la co-construcción del conocimiento. Resulta interesante destacar que este rasgo del manual se replica, pues, al interior de cada uno de los grados, se prefiere utilizar las configuraciones más congruentes: imperativo, en el grado alto, 'ser + adjetivo', en el grado medio y 'verbo obligativo', en el bajo. Cada una de estas configuraciones son recursos que están asociados, desde estudios previos, a la didacticidad y a la divulgación de un conocimiento mucho más especializado (Gläser, 2003; Halliday, 2004a; Gutiérrez, 2007).

Continuando con la analogía entre desarrollo ontogenético y la incorporación al discurso académico, la necesidad de controlar el ambiente dominaría la primera fase de inserción disciplinar, así como domina la primera fase de adquisición de la lengua. En concordancia con ello, en un inicio los roles del intercambio no se configurarían en torno al lenguaje, sino que tan solo en torno al ambiente sociocultural inmediato, es decir, continuarían siendo roles de primer orden, de modo que el aprendiz no está llamado a ser un continuador del diálogo, hecho que marca fuertemente la distancia entre experto y aprendiz que caracteriza el tenor del género manual.

Solo posteriormente, en un estadio más avanzado de la inserción disciplinar, se presentará la necesidad de representar el conocimiento, negociarlo y textualizarlo. Sin embargo, creemos que es necesario que, a medida que el asentamiento del sistema léxico se consolida, se vayan insertando paulatinamente géneros discursivos que comiencen a abrir el espacio para la adquisición de mecanismos de negociación. Esto pone de relieve la necesidad de graduar la complejidad de los textos a los cuales los estudiantes se ven enfrentados. 


\section{CONCLUSIONES}

Los resultados de este estudio permiten sostener que la forma en que se regula la inserción disciplinar a través del manual es directa y fuertemente obligativa, lo que se evidencia en la preferencia del grado alto para construir la obligación en todos los contextos observados. A su vez, esta marcada preferencia se asocia, desde estudios previos, a un discurso didactizante (Halliday, 2004a), divulgativo (Gläser, 2003) y de baja especialización (Gutiérrez, 2007) en el cual el conjunto de conocimientos es presentado de un modo ortodoxo, como algo que no está sujeto a negociación. En este mismo sentido, la relación entre los participantes del intercambio es asimétrica, esto es, de experto a aprendiz. Desde esta primera mirada, se desprende que el manual se presenta como un género que no varía al asociarse a distintas culturas disciplinares (i.e. variable de campo).

La variación por área disciplinar solo comienza a surgir cuando avanzamos en el detalle del análisis y comparamos las probabilidades de ocurrencia de cada configuración dentro de los grados de obligación. A partir de esta segunda mirada, es posible concluir que la ocurrencia de una y otra, en el marco de cada uno de los grados, tiende a coexistir en las Ciencias Sociales y Humanas y a distanciarse en el marco de las Ciencias Básicas y de la Ingeniería, reforzándose, así, la oposición "ciencias blandas" versus "ciencias duras". Creemos que esto estaría dando cuenta de la naturaleza del saber disciplinar, de modo que las Ciencias Básicas y de la Ingeniería se configuran en torno a un contenido disciplinar mucho más dogmático y tecnicalizado que el de las Ciencias Sociales y Humanas, lo que se manifiesta en la distancia que separa la preferencia de configuraciones congruentes de las más metafóricas. En otras palabras, la coexistencia se produciría porque están asociadas a dos distintas movidas retóricas del manual: los ejercicios y las definiciones de conceptos (Parodi, 2008). La no coexistencia, sin embargo, no se debe al predominio de unas de estas movidas retóricas, sino que más bien, al hecho de que el conjunto de conocimientos es, como hemos dicho, más dogmático en las ciencias duras que en las ciencias blandas. Esta última afirmación, por supuesto, es una hipótesis que requiere ser demostrada y, por tanto, constituye una de las proyecciones de esta investigación. A partir de esta idea es posible proyectar una investigación en la que se explore la frecuencia relativa de ocurrencia de otros mecanismos asociados a la especialización, divulgación y didactización; así como una en que se observe el comportamiento del sistema de la obligación en otros géneros utilizados en la enseñanza universitaria. 


\section{REFERENCIAS BIBLIOGRÁFICAS}

Bosque, I. \& Demonte, V. (1999). Gramática descriptiva de la lengua española. Madrid: Espasa-Calpe.

Cademártori, G., Parodi, G. \& Venegas, R. (2007). El discurso escrito y especializado: Las nominalizaciones en los manuales técnicos. En G. Parodi (Ed.), Lingüística de corpus y discursos especializados: Puntos de mira (pp. 79-98). Valparaíso: Ediciones Universitarias de Valparaíso.

Caffarel, A., Martin, J. \& Matthiessen, Ch. (2004). Language typology. A functional perspective. Amsterdam: Benjamins.

Cubo de Severino, L. (2005). Los textos de la ciencia. Buenos Aires: Comunicarte.

Di Tullio, A. (1997). Manual de gramática del español. Desarrollos teóricos. Ejercicios, soluciones. Buenos Aires: Edicial.

Ferrari, S. (2007). La variación de los rasgos de la informatividad y de los tipos de nominalizaciones en los manuales de dos áreas de formación académica. Tesis de magíster, Pontificia Universidad Católica de Valparaíso, Valparaíso, Chile.

Firth, J. (1968). A sinopsis of linguistic theory. En F. Palmer (Ed.), Selected papers of J.R. Firth 1952-59 (pp. 168-205). London: Indiana University Press.

Gläser, R. (2003). A multi-level model for a typology of LSP genres. Fachsprache. Internacional Journal of LSP, 15(1-2), 18-26.

Gutiérrez, R.M. (2007). Oralidad, escritura y especialización: Una caracterización desde el sistema de la modulación. En G. Parodi (Ed.), Lingüística de corpus y discursos especializados: Puntos de mira (pp. 149-178). Valparaíso: Ediciones Universitarias de Valparaíso.

Gutiérrez, R.M. (2008). Metáfora interpersonal en la negociación del conocimiento: Una aproximación al corpus académico PUCV-2006. En G. Parodi, Géneros académicos y géneros profesionales: Accesos discursivos para el saber y el hacer (en prensa). Valparaíso: Ediciones Universitarias de Valparaíso.

Halliday, M. (1982). El lenguaje como semiótica social. La interpretación social del lenguaje y del significado. México, D.F.: Fondo de Cultura Económica.

Halliday, M. (1984). Language as code and language as behaviour: A systemic-functional interpretation of the nature and ontogenesis of dialogue. En R. Fawcett, M. Halliday, S. Lamb \& A. Makkai (Eds.). The semiotics of culture and languague. Vol 1 (pp. 2-35). London: Frances Pinter.

Halliday, M. (2002a). Categories of the theory of grammar (1961). En J. Webster (Ed.), On grammar (pp. 37-94). London: Continuum.

Halliday, M. (2002b). Class in relation to the axes of chain and choice in language (1963). En J. Webster (Ed.), On grammar (pp. 95-105). London: Continuum.

Halliday, M. (2002c). Spoken and written modes of meaning (1987). En J. Webster (Ed.), On grammar (pp. 323-351). London: Continuum. 
Halliday, M. (2004a). Language and konwledge: The 'unpacking' of the text. En J. Webster (Ed.), The Language of the science (pp.24-48). London: Continuum.

Halliday, M. (2004b). The language of early childhood. London: Continuum.

Halliday, M. \& Matthiessen, C. (2004). An introduction to functional grammar. London: Arnold.

Hernández, C. (1986). Gramática funcional del español. Madrid: Gredos.

Hernández, C. (1995). Nueva sintaxis de la lengua española. Salamanca: Ediciones Colegio de España.

Hjelmslev, L. (1974). Prolegómenos a una teoría del lenguaje. Madrid: Gredos.

Hyland, K. (2004). Genre and second language writing. Michigan: University of Michigan.

Ivanic, R. (1998). Writing and identity. The discoursal construction of identity in academic writing. Philadelphia: Benjamins.

Klinge, A. \& Hoeg, H. (2005). Modality. Studies in form and function. London: Equinox.

Marinkovich, J. (2005). Las estrategias de reformulación: El paso desde un texto-fuente a un texto de divulgación didáctica. En G. Parodi (Ed.), Discurso especializado e Instituciones Formadoras (pp. 193-216). Valparaíso: Ediciones Universitarias de Valparaíso.

Marinkovich, J. (2007). La nominalización como recurso de cohesión léxica en los manuales de la formación técnico-profesional. En G. Parodi (Ed.), Lingüística de corpus y discursos especializados: Puntos de mira (pp. 97-114). Valparaíso: Ediciones Universitarias de Valparaíso.

Martin, J. (1992). English text. System and structure. Philadelphia: Benjamins.

Martin, J., Matthiessen, C. \& Painter, C. (1997). Working with functional grammar. London: Arnold.

Matthiessen, C. (1995). Lexicogrammatical cartography: English system. Tokyo: Meadea.

Peronard, M. (1988). El hombre que habla; el hombre que escribe. En Boletín de la Academia Chilena de la Lengua (pp. 59-77). Santiago, Chile.

Palmer, F. (2001). Mood and modality. Cambridge: Cambridge University Press.

Parodi, G. (2008). Pasos retóricos en manuales de cuatro disciplinas. En G. Parodi (Ed.), Géneros académicos y géneros profesionales: Accesos discursivos para el saber y el hacer (en prensa). Valparaíso: Ediciones Universitarias de Valparaíso.

Parodi, G., Venegas, R., Gutiérrez, R.M., Ibáñez, R. (2008). Hacia una definición multidimensional de los géneros del corpus PUCV-2006. En G. Parodi, Géneros académicos y géneros profesionales: Accesos discursivos para el saber y el hacer (en prensa). Valparaíso: Ediciones Universitarias de Valparaíso.

Porto, J. (1991). Del indicativo al subjuntivo: Valores y usos de los modos del verbo. Madrid: Arco Libros.

Rail, S. (2007). Estrategias de reformulación utilizadas por los profesores en los discursos de divul- 
gación científica en contextos de educación infantil. Tesis doctoral, Pontificia Universidad Católica de Valparaíso, Valparaíso, Chile.

Rojo, G. \& Veiga, A. (1999). El tiempo verbal. Los tiempos simples. En I. Bosque \& V. Demonte (Dir.), Gramática descriptiva de la lengua española (pp. 2867-2934). Madrid: Espasa-Calpe.

Swales, J. (2000). Genre analysis. English in academic and research settings. Cambridge: Cambridge University Press. 